\title{
Regional variation in hospitalisation and mortality in heart failure: comparison of England and Lombardy using multistate modelling
}

\author{
Alex Bottle $^{1}$ (D) C Chiara Maria Ventura ${ }^{2} \cdot$ Kumar Dharmarajan $^{3,4} \cdot$ Paul Aylin $^{1}$. \\ Francesca Ieva $^{5}$ - Anna Maria Paganoni ${ }^{2}$
}

Received: 3 July 2016 / Accepted: 3 July 2017 /Published online: 28 July 2017

(C) The Author(s) 2017. This article is an open access publication

\begin{abstract}
Heart failure (HF) is a common, serious chronic condition with high morbidity, hospitalisation and mortality. The healthcare systems of England and the northern Italian region of Lombardy share important similarities and have comprehensive hospital administrative databases linked to the death register. We used them to compare admission for HF and mortality for patients between 2006 and 2012 ( $n=37,185$ for Lombardy, 234,719 for England) with multistate models. Despite close similarities in age, sex and common comorbidities of the two sets of patients, in Lombardy, $\mathrm{HF}$ admissions were longer and more frequent per patient than in England, but short- and medium-term mortality was much lower. English patients had more very short stays, but their very elderly also had longer stays than their Lombardy counterparts. Using a three-state model, the predicted total time
\end{abstract}

Electronic supplementary material The online version of this article (doi:10.1007/s10729-017-9410-x) contains supplementary material, which is available to authorized users.

Alex Bottle

robert.bottle@imperial.ac.uk

1 Dr Foster Unit at Imperial College London, 3 Dorset Rise, London EC4Y 8EN, UK

2 MOX - Modelling and Scientific Computing, Dipartimento di Matematica, Politecnico di Milano, Piazza Leonardo da Vinci, 32, I-20133 Milan, Italy

3 Section of Cardiovascular Medicine, Department of Internal Medicine, Yale School of Medicine, 1 Church Street, Suite 200, New Haven, CT 06510, USA

4 Center for Outcomes Research \& Evaluation, Yale-New Haven Hospital, 1 Church Street, Suite 200, New Haven, CT 06510, USA

5 Dipartimento di Matematica "F.Enriques", Università degli Studi di Milano, via Saldini, 50, I-20133 Milan, Italy spent in hospital showed large differences between the countries: women in England spent an average of 24 days if aged 65 at first admission and 19 days if aged 85; in Lombardy these figures were 68 and 27 days respectively. Eight-state models suggested disease progression that appeared similar in each country. Differences by region within England were modest, with London patients spending more time in hospital and having lower mortality than the rest of England. Whilst clinical practice differences plausibly explain these patterns, we cannot confidently disentangle the impact of alternatives such as coding, casemix, and the availability and use of nonhospital settings. We need to better understand the links between rehospitalisation frequency and mortality.

Keywords Heart failure $\cdot$ Administrative data $\cdot$ Multistate models $\cdot$ International comparison $\cdot$ Mortality $\cdot$ Readmission

\section{Introduction}

According to the Global Burden of Disease study [1], noncommunicable diseases accounted for 23 of the leading 25 causes of years lived with disability worldwide in 2015. One such non-communicable disease, heart failure (HF), affected 40 million people worldwide in 2015; while the agestandardised prevalence rate was little changed from 2005, the numbers of people affected rose by nearly a third in ten years. Heart failure is a common, serious chronic condition with high morbidity and mortality in many countries $[1,2]$. Given its chronic and progressive nature, in many cases it leads to hospitalisation and rehospitalisation [3]. As with other chronic conditions, treating HF is costly and puts increasing pressures on health services around the world. In the USA under the Hospital Readmissions Reduction Program established by Section 3025 of the Affordable Care Act, 
hospitals with high readmission rates for HF and some other conditions incur financial penalties. Hospitalizations represent the main determinants of costs for care of HF patients. With many governments looking to do more with less by learning from overseas, comparisons between countries are increasingly common. In this study, we combine two current projects on the epidemiology and quality of care of HF and set out to compare hospitalisation use in patients with HF in Italy's northern Lombardy region and England.

Before we describe the study's aims, methods and findings, we summarise the main characteristics of each healthcare system and the main potential statistical approaches for modelling multiple admissions in chronic diseases such as HF.

\subsection{Healthcare systems in Lombardy and England}

The two systems have some important similarities: universal coverage backed by the State, a mix of public and private providers with the public ones forming a clear majority, and relatively low healthcare expenditure (both just over $9 \%$ of GDP). England and Italy as a whole have the lowest number of hospital beds relative to population size in Europe, both under 300 per 100,000 inhabitants in 2014 [4]. The Italian government decentralized the country's healthcare system in 1997, giving the regions control over the public money from the central government. Unlike most of the other regions, Lombardy has adopted additional quality standards and set their own reimbursement rates for performance. $22 \%$ of inpatient beds for its ten million citizens are located in private hospitals, either profit or notfor-profit [5]. For England's population of fifty million, comparable information is hard to find, though in the UK as a whole it was estimated that in 2008 private hospitals provided around 11,200 beds for in-hospital stays, with the UK NHS total at 176,679 [4]. 11,200 therefore represents around $6 \%$ of all inpatient beds in the UK. Primary care physicians have a similar gatekeeper role in both administrations and have similar supply, though slightly lower in England: 0.8 per 1000 population in Lombardy in 2007 [6] and 0.7 per 1000 population in England in the same year [new 7].

\subsection{Modelling options}

A number of ways exist to model hospital readmissions. Readmission rates in patients with HF are high and, to try to improve outcomes for patients and reduce costs, incur financial penalties in the USA. These rehospitalisations are usually measured in terms of 30-day all-cause emergency readmissions. However, 30 days is an arbitrary follow-up period and patients are at risk of poor outcomes for longer [8]. In addition, many patients have multiple readmissions, sometimes over a period of several years, so this measure loses information, especially on disease progression [9]. Most studies of readmissions ignore the competing risk of death or account for it by using a combined endpoint of death or readmission [10], which is unsatisfactory, not least because death and readmission are far from being of equal importance. Other modelling options include hurdle models and resource buckets, which can be useful for some questions [11] but are not flexible enough to show progression of the underlying chronic disease. Standard Cox and marginal models are inappropriate for the same reason as they ignore the serial nature of admissions [12]; extensions of these can deal with the clustering of patients within hospitals and multiple events per patient but not the serial nature of those events, or they can account for the competing risk of death under certain assumptions but not the repeat events.

We approach this problem primarily with multistate models, which handle both the competing risk of death and the series of admissions. Multistate models are useful in describing a stochastic process in which a subject at any particular time point occupies one of a few possible states. They have several key features. First, patient states are assumed to be known only at discrete time points - in this study we have daily information. A second is that they employ the Markov assumption that future states depend only on the current state and time, but not on the whole history. This makes the method well suited to chronic disease progression modelling $[12,13]$. How likely the patient is to change state at any time $t$ is described by the transition intensity, which may depend on time $t$ and on a set of individual and time-dependent variables. The transition intensities can easily be estimated taking into account the effect of covariates. Once we know the intensities, we gain the more interpretable probability of transition under the Markov assumption. These probabilities refer to whether each state will be the next one - given that the patient changes state. Compared with regression or survival analysis, these models are less familiar as they are not generally taught on statistics Master's courses and because implementation in standard software is either not possible or not as straightforward as logistic regression, for instance. To date there have been only a few studies using multistate models in heart failure, each limited to a small sample [14] or one country [12, 15]. For context, we also use publicly available population estimates to calculate admission rates and provide estimated prevalences.

\subsection{Aims of the study}

We set out to compare hospitalisation use in patients with HF in Italy's Lombardy region and England. Specifically, we compare the unplanned use of inpatient hospital beds for HF and mortality following a first HF admission in Lombardy and England both overall and by age and sex. Both areas' administrative data sets have population coverage and linkage to 
national death registers and thereby capture deaths both in and out of hospital.

Given that the population of England is five times that of Lombardy and that OECD research found that regional differences in quality of care and outcomes can be considerable, including in cardiology within Italy [16], we also split England into its ten government regions (we used the former Strategic Health Authorities that existed during our study data period).

\section{Methods}

\subsection{Data source and definition of cohort}

Administrative databases were used for both countries. For England, this was Hospital Episodes Statistics (HES), which covers all patients admitted to public (NHS) hospitals nationally (see the NHS Digital website). Each record is a finished consultant episode, which covers the continuous period of time during which a patient is under the care of a consultant (senior doctor) or allied health professional: an admission can comprise more than one episode. Episodes were linked into admissions, with transfers to other hospitals combined to avoid multiple-counting. Hospitalizations represent the observable part of the latent degenerative disease process, and we therefore examined the set of admissions with a principal diagnosis of HF. These were defined as those with a primary diagnosis of HF (ICD10 I50); only people aged 18 or over and emergency (unplanned) admissions were included. These were extracted for the period April 2006 to March 2012; we tracked back three years and excluded patients with other HF admissions during that period in order to ensure that a set of index (first) HF admissions were analysed to better capture an extended course of disease progression. HES data are linked to the national death registry held by the Office for National Statistics to capture the fact and date of death if it did not occur in hospital. Region was defined as the former ten Strategic Health Authorities (SHAs) within England on the basis of the location of the hospital as per the HES Online website. The ten SHAs were established on 1 July 2006 and abolished on 31 March 2013, which covers our data period. They were responsible for implementing Department of Health policy and overseeing the local organisations that ran or commissioned NHS services within their region.

A similar process was followed to extract admissions for HF in Lombardy. The HF extract used in this study was part of a bigger dataset of all patients admitted in the region selecting patients with incident events between January 2006 and December 2012. For a detailed description of the extraction criteria to construct the data set of interest see Mazzali et al. [17]. In brief, the Lombardy project's aims are to study the epidemiology, short- and medium-term outcomes and the variations in processes of care for patients hospitalized for HF.

The maximum follow-up length was reduced slightly and set equal to that in the English data set (2216 days, or 6.1 years) to help make a fair comparison. 692 patients had events censored this way.

Records in both countries were excluded if they had missing or invalid age, sex, length of stay, admission dates or if the same patient had two or more admissions with overlapping admission and discharge dates. For each patient, the time counter began at time $t=0$ on the day of index admission. Readmissions on the same day as previous discharge were included, with half a day added to $t$ to allow inclusion in the multistate models. Similarly, same-day discharges were assumed to last 0.5 days. Transfers between hospitals can be reliably identified within the data set and were not counted as readmissions.

\subsection{Statistical methods}

Patient characteristics were compared by country using descriptive statistics. The length of the hospital stay (LOS) for each admission was summarised using histograms. In-hospital mortality for each $\mathrm{HF}$ admission and total crude mortality (i.e. deaths in and out of hospital combined) was compared using Kaplan-Meier survival plots.

Two types of multistate models were implemented: one with just three states (alive in hospital, alive out of hospital, dead) and one with eight states that accommodated up to each patient's first four HF admissions. In the second one, all admissions greater than the fourth were combined. Data were prepared using SAS and analysed using the " $\mathrm{msm}$ " package within R [18]; the development of "msm" was motivated by disease modelling applications. This package provides a set of useful functions to describe features of Markov process: intensity matrices, transition probability matrices, mean sojourn times (e.g. mean time spent in hospital), probability that each state is next, total period of time spent in a state (e.g. total time spent in hospital) and others. We report results from "totlos.msm" (total time spent in each state, both for all patients combined as an average and for two selected combinations of age and sex) and "sojourn.msm" (the "sojourn time" or mean time spent in each state: we give this both overall and for the same selected combinations of age and sex). These can be used to describe the time spent in hospital - either total time with three states or the time in the first, second and third admission separately with eight states - and the time alive in the community between successive admissions or as expected survival time. They can be calculated for a set period of follow-up, e.g. five years, or for infinite follow-up, i.e. for the patient's whole remaining lifetime - we chose the former option. The "total stay" measures such as total time spent in hospital are not applicable for the eight-state model because, 
with each admission counted separately, there are no reverse transitions.

Multistate models were fitted firstly as crude without covariates and secondly with age and sex as covariates. The latter allowed sojourn and predicted survival times to be calculated by age and sex: for illustration, we present results for ages 65 and 85 years, representing the lower boundary of common definitions of 'elderly' (65) and very elderly (85). Lastly, we included some common comorbidities as extra covariates and report their hazard ratios. All models were run on each country's data set separately.

Model assumptions were checked graphically and statistically. The relation between in-hospital mortality and length of stay was investigated using Kaplan-Meier plots for each HF admission, and the hazard was found to be constant. The proportionality assumption for hazard ratios for covariate effects was checked visually by Kaplan-Meier plots and formally by including an interaction with time and testing its significance; violations of this assumption were found to be insignificant. The msm package allows for an informal check of the Markovian assumption via the plot.prevalence.msm function, and the resulting plots showed good agreement between the observed and predicted outcomes for each state. More formally, we tested the association between the time spent in the previous state and the probability of transition from the current state as per Section 4.1.2 of [19] via hazard ratios and their $95 \%$ confidence intervals from the hazard.msm function. For example, in the three-state models we tested whether the number of days spent as an inpatient was associated with the transition from the community to death or with the transition from the community back to the hospital. All the resulting hazard ratios were between 0.993 and 1.008, indicating no appreciable violation of the Markov assumption (Online Resource 1).

\section{Results}

\subsection{Prevalence, admission and mortality rates}

In Lombardy, the Utilization of Regional Health Service Databases project produced admission and prevalence rate estimates for each year to 2012. In 2006, the first data year of this present study, there were 128,697 patients with diagnosed HF in a population of $9,475,202$, for a prevalence of $1.4 \%$; in 2012 , the estimated prevalence was $1.7 \%$ (1.8\% in men and $1.6 \%$ in women). In the UK, the only national prevalence data come from primary care registers created by general practitioners (GPs) used in the national pay-forperformance scheme called the Quality and Outcomes Framework (QOF). In 2012/13, the HF register from QOF recorded a prevalence of $0.7 \%$ for England as a whole, ranging from $0.5 \%$ in London to $0.9 \%$ in the North East government region [20]. An analysis of primary care records from a roughly $10 \%$ sample of GPs in England known as the Clinical Practice Research Datalink gave a higher estimated prevalence for 2013 of $1.0 \%$ ( $1.2 \%$ for men and $0.8 \%$ for women) [20]. While higher than the QOF estimate, this was still appreciably lower than the figure from the Lombardy project.

\subsection{Descriptive statistics}

Less than $0.1 \%$ of records were excluded due to missing data in England; in Lombardy this was $<2 \%$. In our Lombardy data set there were a total of 37,185 patients with 22,519 deaths $(60.6 \%)$ after a mean follow-up of 1.4 years (median 0.9 years). In England, there were 234,719 patients with 130,916 deaths $(55.8 \%)$ after a mean follow-up of 1.6 years (median 0.7 years). The maximum follow-up was 6.2 years. 70 English patients $(0.03 \%)$ could not be matched to a region and were excluded. Table 1 summarises the number of admissions for HF per patient, which was higher on average in Lombardy. More than 2 in 5 patients in Lombardy but fewer than 1 in 5 patients in England had more than one emergency HF admission during the follow-up period. Table 2 describes the patient characteristics and short-term crude outcomes, which shows similar age, sex and comorbidity distributions in each country, except for a higher prevalence of hypertension recorded in England and higher prevalence of diabetes with long-term complications recorded in Lombardy. Diabetes without complications was not available for the Lombardy data set. Following the index HF admission, Lombardy's 30day readmission rate was similar to England's, but its one-year mortality was much lower even though, as noted above, the overall proportion of patients who died was higher in Lombardy.

Figure 1 is the Kaplan-Meier plot for survival in each country, which shows that Lombardy gains a survival advantage very early that it relinquishes only after approximately 1700 days of follow-up since index admission.

The LOS patterns were markedly different for Lombardy and England, with much more minor variations between each patient's first, second and third HF admission (Figs. 2 and 3). Five percent of England's admissions were same-day

Table 1 Distribution of number of admissions to hospital between HF patients in Lombardy and England and related percentages

\begin{tabular}{llrlr}
\hline $\begin{array}{l}\text { Number of HF } \\
\text { adms per patient }\end{array}$ & $\begin{array}{l}\text { Number of } \\
\text { patients } \\
\text { (Lombardy) }\end{array}$ & $\begin{array}{l}\text { \% of } \\
\text { total }\end{array}$ & $\begin{array}{l}\text { Number of } \\
\text { patients } \\
\text { (England) }\end{array}$ & $\begin{array}{l}\% \text { of } \\
\text { total }\end{array}$ \\
\hline 1 & 21,908 & 58.9 & 191,355 & 81.5 \\
2 & 7872 & 21.2 & 30,713 & 13.1 \\
3 & 3502 & 9.4 & 8023 & 3.4 \\
4 & 1702 & 4.6 & 2669 & 1.2 \\
$5+$ & 2201 & 5.9 & 1960 & 0.8 \\
\hline
\end{tabular}


Table 2 Patient characteristics and overall crude outcome rates in Lombardy and England

\begin{tabular}{llllr}
\hline Factor* & Lombardy N & \% of total & England N & \% of total \\
\hline Age: mean (SD) & $78.3(11.8)$ & $\mathrm{n} / \mathrm{a}$ & $78.5(11.7)$ & $\mathrm{n} / \mathrm{a}$ \\
Males & 17,645 & 47.5 & 116,949 & 49.8 \\
Females & 19,540 & 52.5 & 117,770 & 50.2 \\
Hypertension & 16,180 & 43.5 & 114,447 & 48.8 \\
Diabetes mellitus without complications & $\mathrm{n} / \mathrm{a}$ & $\mathrm{n} / \mathrm{a}$ & 60,202 & 25.6 \\
Complications of diabetes & 2847 & 7.7 & 6059 & 2.6 \\
Chronic pulmonary disease & 8992 & 24.2 & 51,454 & 21.9 \\
Arrhythmia & 17,675 & 47.5 & 116,887 & 49.8 \\
Dementia & 1987 & 5.3 & 10,240 & 4.4 \\
Renal disease & 7843 & 21.1 & 52,296 & 22.3 \\
Crude outcomes* & & & & \\
$\quad$ 30-day readmission after index & 2122 & 6.2 & 11,699 & 6.0 \\
365-day mortality after index & 9858 & 26.5 & 92,335 & 39.3 \\
\hline
\end{tabular}

* All comparisons had $p<0.01$ except for 30-day readmission rates, $p=0.055$ discharges, whereas every Lombardy patient stayed at least one night. The Lombardy distribution was noticeably less skewed than the English one. When all patients are combined, the preponderance of short stays in England leads to a lower quartile, median and mean that are lower in England than in Lombardy, whereas the upper quartile and higher percentiles are similar in both (Table 3). When the results are split by age, however, whereas the picture for the 65-year-olds matches that for all patients combined, the upper quartile and higher percentiles for stays by patients aged 85 years are higher in England than in Lombardy, meaning that their distribution is stretched: England's very elderly short-stay patients stay less time than their Lombardy counterparts whereas its very elderly long-stay patients stay more time.

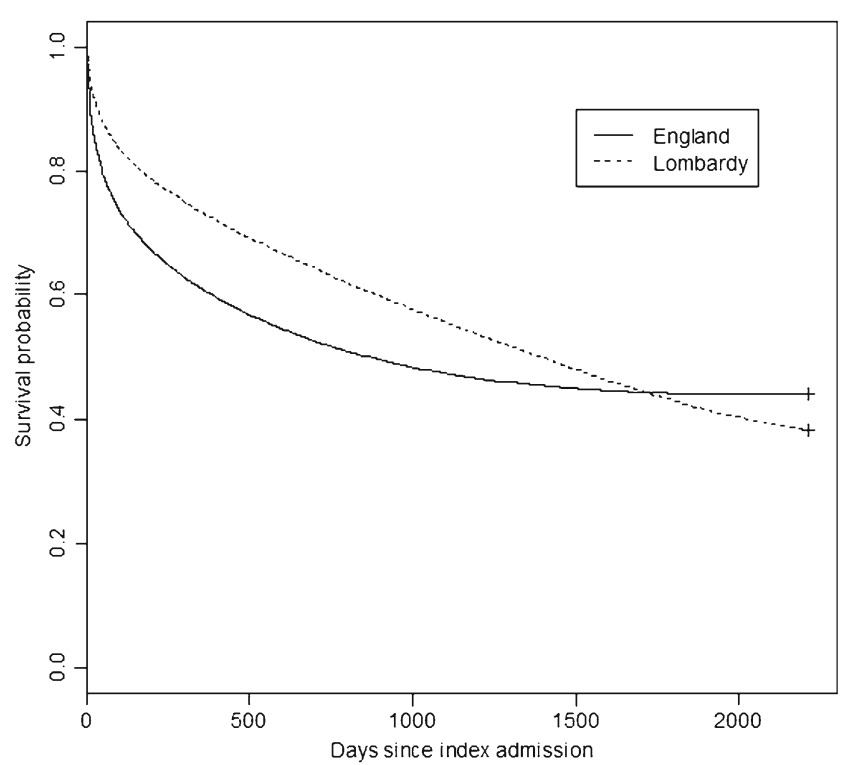

Fig. 1 Kaplan-Meier plot for the proportion of patients alive from time since first HF admission in each country

\subsection{Results for three-state models}

The mean times spent in each state for the three-state model with age and sex were: 14.0 days alive in hospital $(95 \%$ confidence interval (CI) 13.9-14.1) for Lombardy, 12.3 days (CI 12.2-12.3) for England; 459.6 days alive out of hospital (CI 455.3-463.9) for Lombardy and 813.0 days (CI 808.7-817.3) for England. The Figures 14.0 and 12.4 are average lengths of stay in hospital averaged across both patients and admissions and so represent how many days a patient can expect to stay each time they admitted for HF (see Table 4).

For the three-state model with age and sex, the average total time spent in hospital per patient when summed across all the HF admissions that each patient had was 37.4 days for Lombardy and 20.0 days for England. These figures were very similar for the crude model (19.4 days for England, Table 4). On average, Lombardy patients could expect to spend nearly twice as many days in total in hospital for HF than English ones. This is a better measure of the burden of disease in terms of hospitalisation and could be converted into a total monetary cost for economic analysis.

Multistate models also give the estimated probability that each state will be the next one, given that the patient changes state. For a three-state model, if the patient is in hospital, the two possible transitions are live discharge or death; if the patient is alive in the community, the two possible transitions are readmission or death. When patients in hospital changed state in Lombardy, they had a 9\% probability of dying while in hospital; in England this was 16\% overall, ranging from 13\% in London to $18 \%$ in the North West, East of England and South West SHAs. When patients alive in the community changed state, readmission was twice as likely as death in Lombardy (66\% compared with 34\%) but less likely than death in England (44\% compared with 56\%). Among English regions, the lowest chance of transitioning to death 
Fig. 2 Length of stay distribution for each patient's first three HF admissions, Lombardy, with highest $1 \%$ outliers excluded

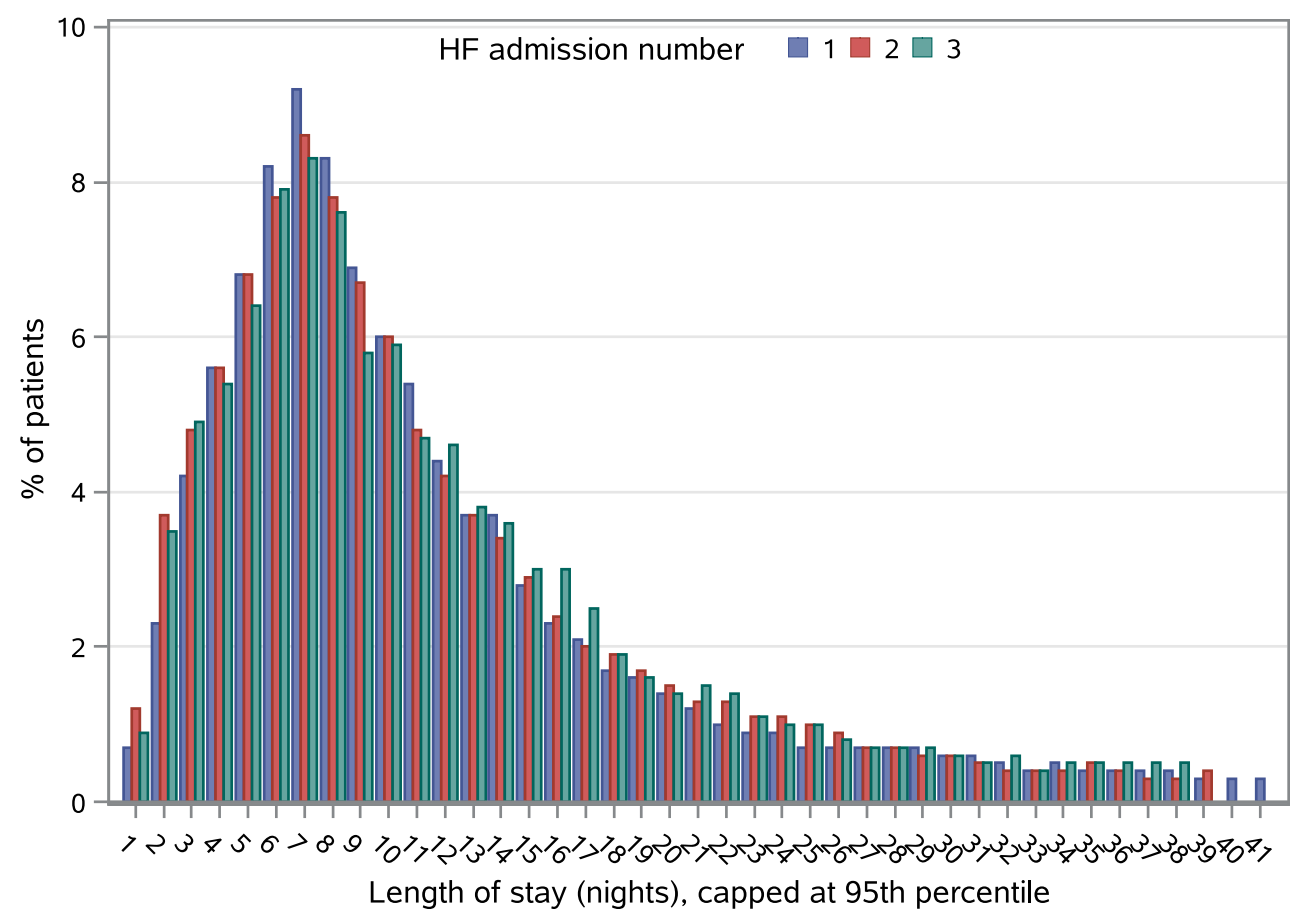

while in the community was also lowest (50\%) in London and highest in the South West (61\%, Online Resource 2).

Table 5 gives the expected time spent in hospital by region for the same age-sex combinations. Here, the differences between Lombardy and England were large, with 65-year-olds spending an average of 68 days in a Lombardy hospital but only 23 or 24 days in an English one during the follow-up period; for 85-year-olds, the difference was just over a week. All these differences were statistically significant at the $5 \%$ level as their confidence intervals from msm did not overlap. Within England, the shortest time spent in hospital was in the East Of England region, with the longest times spent in London for both ages and sexes. Restricting the set of patients by removing those with the $1 \%$ longest stays had only a modest effect on the estimates, reducing them by less than $10 \%$, and did not change the patterns.

Table 6 gives the expected time alive and out of hospital for males and females aged 65 and 85 years during one and five
Fig. 3 Length of stay distribution for each patient's first three HF admissions, England, with highest $1 \%$ outliers excluded

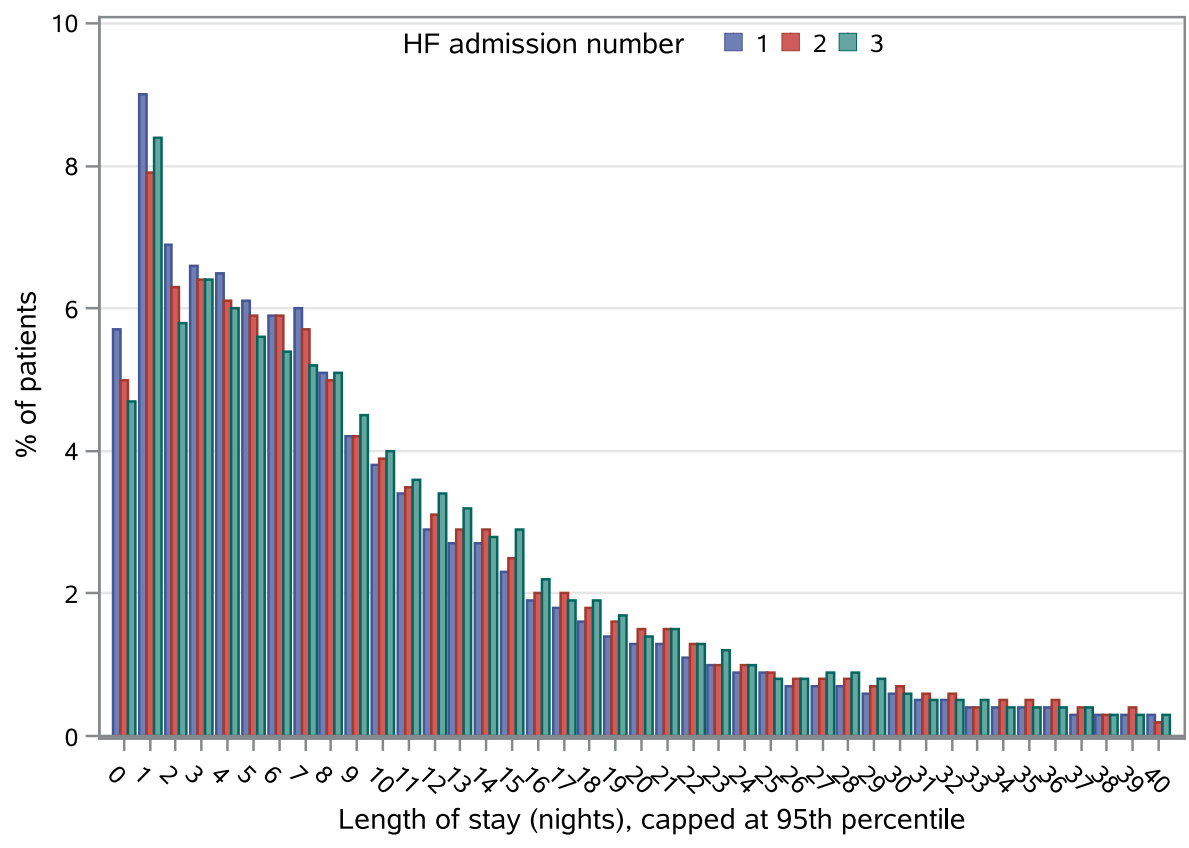


Table 3 Summary crude statistics for length of stay for all HF admissions combined for Lombardy vs England

\begin{tabular}{|c|c|c|c|c|c|c|}
\hline Age-sex group & $\begin{array}{l}\text { Number of } \\
\text { patients }\end{array}$ & $\begin{array}{l}\text { Lower } \\
\text { quartile }\end{array}$ & Median & Mean & $\begin{array}{l}\text { Upper } \\
\text { quartile }\end{array}$ & 90th percentile \\
\hline \multicolumn{7}{|l|}{ All patients: } \\
\hline Lombardy & 37,185 & 7 & 10 & 14.0 & 16.2 & 27.5 \\
\hline England & 234,719 & 4 & 8 & 12.5 & 16 & 28 \\
\hline \multicolumn{7}{|c|}{ 65-yr.-old males: } \\
\hline Lombardy & 333 & 6 & 10 & 14.2 & 18 & 30 \\
\hline England & 1678 & 3 & 7 & 10.3 & 13 & 22 \\
\hline \multicolumn{7}{|c|}{ 65-yr.-old females: } \\
\hline Lombardy & 165 & 6 & 10 & 15.7 & 19 & 32 \\
\hline England & 894 & 3 & 7 & 11.5 & 14 & 26.3 \\
\hline \multicolumn{7}{|c|}{ 85-yr.-old males: } \\
\hline Lombardy & 1039 & 7 & 10 & 12.9 & 15 & 23 \\
\hline England & 4642 & 4 & 9 & 13.3 & 17 & 30 \\
\hline \multicolumn{7}{|c|}{ 85-yr.-old females: } \\
\hline Lombardy & 556 & 7 & 10 & 12.9 & 15.5 & 24 \\
\hline England & 5765 & 4 & 8.5 & 13.4 & 17 & 30 \\
\hline
\end{tabular}

years following live discharge from the index HF admission. These always favoured Lombardy over England and females over males. Within England, London had the longest expected time alive and out of hospital for both ages and sexes. Taken together, Fig. 1, Tables 5 and 6 show that Lombardy patients spend more time on average in hospital but also more time alive in the first one and five years after index discharge than their English counterparts. Lombardy's mortality only overtakes that of England later (Fig. 1).

Lastly for the 3-state model, Table 7 gives the transitionspecific hazard ratios (HRs) for age, sex and the five most common comorbidities. As in regular survival analysis, hazards from multistate models estimate the probability of the outcome after a given number of days given that the patient has not already had the outcome before that time; HRs compare these probabilities for one gender relative to the other, for instance, or for a unit increase in age. Let's consider renal disease as an example. We can see that in both Lombardy and England it is associated with a lower hazard for live discharge and a higher hazard of death in hospital, readmission and death in the community. The HRs are above 1 for the latter three outcomes, indicating higher risk of these outcomes than for patients without renal disease. HRs for live discharge that exceed 1 , however, reflect a shorter length of

Table 4 Number of days spent in hospital by region, three-state model without covariates

\begin{tabular}{lccc}
\hline Region & Number of patients & $\begin{array}{l}\text { Mean days in hospital per } \\
\text { patient on average } \\
(95 \% \text { CI) }\end{array}$ & $\begin{array}{l}\text { Total days in hospital per } \\
\text { patient on average }\end{array}$ \\
\hline Lombardy & 37,185 & $14.0(13.9-14.1)$ & 37.4 \\
All England & 234,649 & $12.3(12.2-12.3)$ & 19.4 \\
North East Strategic Health Authority & 13,523 & $12.3(12.1-12.5)$ & 19.7 \\
North West Strategic Health Authority & 34,627 & $12.4(12.2-12.5)$ & 19.3 \\
Yorkshire and The Humber Strategic Health Authority & 26,154 & $12.4(12.2-12.5)$ & 19.3 \\
East Midlands Strategic Health Authority & 19,323 & $12.8(12.7-13.0)$ & 20.4 \\
West Midlands Strategic Health Authority & 28,260 & $11.8(11.7-11.9)$ & 19.3 \\
East Of England Strategic Health Authority & 25,457 & $11.5(11.4-11.6)$ & 17.6 \\
London Strategic Health Authority & 30,202 & $12.2(12.1-12.3)$ & 21.4 \\
South East Coast Strategic Health Authority & 18,759 & $11.7(11.5-11.8)$ & 17.8 \\
South Central Strategic Health Authority & 13,552 & $12.4(12.2-12.5)$ & 18.6 \\
South West Strategic Health Authority & 24,792 & $13.4(13.2-13.5)$ & 19.7 \\
\hline
\end{tabular}


Table 5 Predicted total days spent in hospital during the years of follow-up, by region for selected ages and sex (figures in brackets are from models without longest-staying $1 \%$ of admissions)

\begin{tabular}{|c|c|c|c|c|}
\hline Region & Age 65 , male & Age 65 , female & Age 85 , male & Age 85 , female \\
\hline Lombardy & $68.0(62.4)$ & $68.5(63.3)$ & $26.5(25.4)$ & $27.1(26.0)$ \\
\hline All England & $22.7(21.2)$ & $23.8(21.7)$ & $18.1(17.0)$ & $19.2(17.6)$ \\
\hline North East Strategic Health Authority & $22.1(20.8)$ & $22.6(20.6)$ & $18.6(17.7)$ & $19.4(17.9)$ \\
\hline North West Strategic Health Authority & $21.7(20.2)$ & $22.3(20.5)$ & $18.3(16.9)$ & $19.0(17.4)$ \\
\hline Yorkshire and The Humber Strategic Health Authority & $21.6(20.5)$ & $23.0(21.2)$ & $18.0(17.3)$ & $19.3(18.0)$ \\
\hline East Midlands Strategic Health Authority & $23.8(21.8)$ & $25.1(22.7)$ & $19.1(17.7)$ & $20.2(18.5)$ \\
\hline West Midlands Strategic Health Authority & $22.0(20.4)$ & $23.0(21.2)$ & $18.1(16.8)$ & $19.0(17.5)$ \\
\hline East Of England Strategic Health Authority & $21.4(20.3)$ & $22.3(20.8)$ & $16.6(15.8)$ & $17.5(16.3)$ \\
\hline London Strategic Health Authority & $26.1(24.0)$ & $27.2(24.8)$ & $19.7(18.4)$ & $20.5(19.0)$ \\
\hline South East Coast Strategic Health Authority & $21.0(20.0)$ & $21.0(19.3)$ & $17.2(16.2)$ & $17.6(16.1)$ \\
\hline South Central Strategic Health Authority & $21.7(19.3)$ & $23.6(20.4)$ & $17.1(15.9)$ & $18.6(16.9)$ \\
\hline South West Strategic Health Authority & $22.9(21.1)$ & $24.3(21.9)$ & $18.5(17.1)$ & $19.9(17.9)$ \\
\hline
\end{tabular}

hospital stay, and we can therefore see that renal disease is associated with longer stays. Females in either country have lower readmission and death rates than males but longer hospital stays; these longer stays are compatible with the finding on mean predicted sojourn times for being in hospital.

\subsection{Results for the eight-state model}

For the eight-state model, there was little difference in either mean or total time spent in or out of hospital when either the crude model was used or when the model included age and sex. Table 8 gives the mean time spent in and out of hospital.

The probabilities that each state will be the next one, given that the patient changes state, differed by country but showed the same pattern for each HF admission (Table 9). The probability of dying in hospital was $8 \%-9 \%$ for the first three admissions in Lombardy and $16 \%-17 \%$ for the first three admissions in England. The chance of death following live discharge progressively fell after each admission in each country: $40 \%, 32 \%$ and $28 \%$ after the first, second and third HF discharge respectively in Lombardy compared with 60\%, 48\% and 40\% respectively in England.

\section{Discussion}

\subsection{Main findings}

We used descriptive statistics and multistate models to compare hospital inpatient use for heart failure and mortality between England and the Lombardy region in northern Italy. Although the distributions of age, sex and the main measured comorbidities of the admitted patients were rather similar between the countries, some notable differences emerged.

Table 6 Predicted number of days spent alive and out of hospital in the one and five years following their index HF admission by region for selected ages and sex, for the two countries (five-year figures in brackets)

\begin{tabular}{|c|c|c|c|c|}
\hline Region & Age 65 , male & Age 65 , female & Age 85 , male & Age 85, female \\
\hline Lombardy & $299(1117)$ & $304(1172)$ & $248(642)$ & $257(703)$ \\
\hline All England & $237(512)$ & $243(549)$ & $188(322)$ & $195(350)$ \\
\hline North East Strategic Health Authority & $241(520)$ & $242(529)$ & $195(341)$ & $196(349)$ \\
\hline North West Strategic Health Authority & $232(488)$ & $237(519)$ & $181(310)$ & $188(333)$ \\
\hline Yorkshire and The Humber Strategic Health Authority & $232(483)$ & $238(519)$ & $187(322)$ & $194(350)$ \\
\hline East Midlands Strategic Health Authority & $237(519)$ & $242(546)$ & $190(333)$ & $196(355)$ \\
\hline West Midlands Strategic Health Authority & $239(518)$ & $244(552)$ & $188(325)$ & $194(350)$ \\
\hline East Of England Strategic Health Authority & $236(507)$ & $243(549)$ & $181(303)$ & $189(332)$ \\
\hline London Strategic Health Authority & $247(558)$ & $251(586)$ & $201(362)$ & $205(384)$ \\
\hline South East Coast Strategic Health Authority & $239(508)$ & $249(563)$ & $184(302)$ & $196(343)$ \\
\hline South Central Strategic Health Authority & $236(506)$ & $243(548)$ & $187(318)$ & $195(351)$ \\
\hline South West Strategic Health Authority & $235(500)$ & $244(557)$ & $184(312)$ & $195(355)$ \\
\hline
\end{tabular}


Table 7 Hazard ratios (95\% CI) for age, sex and the five most common comorbidities in Lombardy and England in the 3-state model

\begin{tabular}{lllll}
\hline Region and Factor & Live discharge & Death in hospital & Readmission & Death out of hospital \\
\hline Lombardy & & & & \\
$\quad$ Age (per year) & $1.004(1.003-1.004)$ & $1.06(1.06-1.06)$ & $0.98(0.98-0.99)$ & $1.05(1.04-1.05)$ \\
Female sex & $0.98(0.96-0.99)$ & $0.86(0.82-0.90)$ & $0.95(0.93-0.97)$ & $0.96(0.93-0.99)$ \\
Renal disease & $0.85(0.83-0.86)$ & $1.15(1.09-1.22)$ & $1.50(1.46-1.54)$ & $1.35(1.30-1.40)$ \\
Arrhythmias & $0.99(0.97-1.00)$ & $0.77(0.73-0.81)$ & $1.23(1.20-1.26)$ & $0.97(0.94-0.995)$ \\
Chronic pulmonary disease & $0.90(0.89-0.92)$ & $0.97(0.92-1.03)$ & $1.31(1.28-1.34)$ & $1.12(1.08-1.16)$ \\
Diabetes with complications & $0.93(0.90-0.96)$ & $1.02(0.93-1.12)$ & $1.26(1.22-1.31)$ & $1.26(1.19-1.34)$ \\
Hypertension & $0.98(0.96-0.99)$ & $0.69(0.66-0.73)$ & $1.08(1.06-1.11)$ & $0.88(0.85-0.91)$ \\
England & & & & $1.01(1.01-1.01)$ \\
Age (per year) & $0.99(0.99-0.99)$ & $1.03(1.03-1.03)$ & $0.85(0.84-0.87)$ & $0.87(0.85-0.88)$ \\
Female sex & $0.92(0.91-0.93)$ & $0.94(0.92-0.95)$ & $1.87(1.83-1.90)$ & $1.63(1.61-1.66)$ \\
Renal disease & $0.71(0.70-0.71)$ & $1.42(1.39-1.45)$ & $1.31(1.29-1.33)$ & $1.07(1.06-1.09)$ \\
Arrhythmias & $0.94(0.93-0.95)$ & $0.89(0.88-0.91)$ & $1.26(1.24-1.29)$ & $1.28(1.25-1.30)$ \\
Chronic pulmonary disease & $0.99(0.98-1.00)$ & $1.03(1.01-1.05)$ & $1.35(1.30-1.41)$ & $1.30(1.25-1.36)$ \\
Diabetes with complications & $0.89(0.86-0.91)$ & $0.84(0.79-0.89)$ & $1.11(1.09-1.13)$ & $0.84(0.82-0.85)$ \\
Hypertension & $1.14(1.14-1.15)$ & $0.82(0.80-0.83)$ &
\end{tabular}

Lombardy patients had more HF admissions per person and spent more time in hospital both per admission and in total than those in England, with the mean and median stays for England driven by the substantial subset of very short stays there. Summary inpatient length of stay statistics by age revealed the widest spread for patients aged 85 in England, whose lower quartile was lower and whose upper quartile was higher than for their Lombardy counterparts. The crude mortality for Lombardy patients was much lower early on than in England and remained lower until nearly five years after the first $\mathrm{HF}$ admission.

Using the three-state model, the predicted total time spent in hospital over the follow-up period by age and sex also showed large differences between the countries: for example, in England women spent an average of 24 days if aged 65 at first admission and 19 days if aged 85, and in Lombardy women spent an average of 68 days in hospital if aged 65 , falling to 27 days if aged 85 . These findings suggest that Lombardy's patterns differed much more from England's patterns than any of England's regions differed from each other: patients in the London region stayed the longest in hospital but had the lowest mortality. The patterns were not affected by outliers. Taken together, the results show that as well as spending more time in hospital, Lombardy's patients spent much more time alive and out of hospital than those in England because their survival advantage was maintained for most of the follow-up period.

Extending the model to eight states allowed us to examine the sojourn times and transition probabilities for each of the first three HF admissions separately. This showed that in both countries the probability of transition to death whilst in hospital was the same during each of the patient's first three HF admissions (and constant for each day since admission) and that the probability of transition to death whilst in the community progressively fell after each of the patient's first three HF admissions. The gap between successive admissions fell with each admission by a similar proportional amount in both Lombardy and England, which suggests similar rates of HF progression.

Whilst the healthcare systems in the two countries have some important similarities as outlined in the Introduction, our analysis of HF outcomes suggests notable differences in
Table 8 Mean time spent in days in each state for 8-state model, no covariates

\begin{tabular}{lclll}
\hline State & Lombardy mean & $95 \%$ CI & England mean & $95 \%$ CI \\
\hline Admission 1 & 14.0 & $13.9-14.2$ & 12.2 & $12.1-12.2$ \\
Admission 2 & 13.6 & $13.3-13.8$ & 12.7 & $12.6-12.8$ \\
Admission 3 & 13.9 & $13.6-14.2$ & 12.8 & $12.6-13.0$ \\
Alive after discharge 1 & 591 & $584-598$ & 933 & $928-939$ \\
Alive after discharge 2 & 395 & $388-403$ & 516 & $510-523$ \\
Alive after discharge 3 & 298 & $291-306$ & 365 & $357-374$ \\
\hline
\end{tabular}


Table 9 Probability that each state will be the next one given that the patient changes state from the 8-state model

\begin{tabular}{|c|c|c|c|c|c|c|c|c|}
\hline State & Adm 1 & Dis 1 & Adm 2 & Dis 2 & Adm 3 & Dis 3 & Adm 4+ & Death \\
\hline \multicolumn{9}{|c|}{ Lombardy } \\
\hline Adm 1 & & 0.92 & & & & & & 0.08 \\
\hline Dis 1 & & & 0.60 & & & & & 0.40 \\
\hline Adm 2 & & & & 0.91 & & & & 0.09 \\
\hline Dis 2 & & & & & 0.68 & & & 0.32 \\
\hline Adm 3 & & & & & & 0.91 & & 0.09 \\
\hline Dis 3 & & & & & & & 0.72 & 0.28 \\
\hline Adm 4+ & & & & & & & & 1 \\
\hline \multicolumn{9}{|l|}{ England } \\
\hline Adm 1 & & 0.84 & & & & & & 0.16 \\
\hline Dis 1 & & & 0.40 & & & & & 0.60 \\
\hline Adm 2 & & & & 0.83 & & & & 0.17 \\
\hline Dis 2 & & & & & 0.52 & & & 0.48 \\
\hline Adm 3 & & & & & & 0.84 & & 0.16 \\
\hline Dis 3 & & & & & & & 0.60 & 0.40 \\
\hline Adm 4+ & & & & & & & & 1 \\
\hline
\end{tabular}

The $95 \%$ CIs in this table are very narrow (at most $1 \%$ either side of the point estimates) and have therefore been omitted

Each patient's fourth and subsequent admissions have been combined so that the only possible change of state is death, hence the probability of 1 systems and/or clinical management of these patients, which we now discuss.

\subsection{Potential explanations for these findings}

In this section, we first consider artefactual explanations before discussing what impact differences in quality of care could have had.

\subsubsection{Lombardy's death rate is consistently lower in hospital and lower in England overall for most of the follow-up period}

Coverage of the national and regional death registers is very high in both areas, and, whilst linkage with the hospital admissions databases is not $100 \%$ successful it is also very good, so this will not explain the differences. Instead, this finding could be partly due to casemix if English patients are sicker at each admission than in Lombardy. There are at least three arguments against this explanation. First, the demographics and the prevalence of the main comorbidities were similar in both, though we lacked information on symptom severity, frailty and other casemix factors that could have differed; results from the European Society of Cardiology HF Registry indicate appreciable regional variations in outcomes and patient characteristics beyond demographics such as aetiology and ejection fraction [21]. The estimated HF prevalence in Lombardy was up to twice that in England, which could be due to many reasons: perhaps there is more underdiagnosis of HF in England than in Lombardy so that the patients who do get an HF diagnosis are sicker. Second, the time between successive admissions shortened by a similar relative amount in each country, suggesting that the HF cohorts deteriorated at a similar rate in each country (see Table 8 ). Third, one would expect sicker patients to go on to have more admissions than less-sick ones, which takes us to our next point.

\subsubsection{Lombardy patients had more HF admissions each on average than English ones}

This could happen due to coding in two ways. First, some Lombardy HF admissions might actually have been for nonHF diagnoses and so should have been excluded from our cohort. The extraction criteria described in [17] are less restrictive than used in England, so this is possible to an extent. Second, some England non-HF admissions might actually have been for HF and so should have been included in our cohort. However, this is not likely to have a big effect, as the primary diagnosis in English data is well recorded [22].

This difference could also happen if those who survive their first HF admission in Lombardy are sicker than those who survive it in England. The probability of transitioning to death from alive in hospital was $16 \%$ in England but only $9 \%$ in Lombardy, so this is possible. This would argue against the theory raised earlier than English HF patients are sicker on admission than Lombardy ones, as this would imply an even greater difference in mortality between the countries.

Another potential scenario is that index admission survivors are equally sick in the two countries but the admission 
threshold is lower in Lombardy. Such a higher willingness and capacity to (re)admit these patients could also help explain the in-hospital mortality differences between the countries in all of the first three HF admissions (seen in the eight-state model).

\subsubsection{Lombardy patients spent more days in hospital in total for HF than those in England}

This is due to a combination of having more admissions per patient, as just discussed, having higher mean lengths of stay per admission, and better survival for most of the follow-up period. The higher mean stays could again be due to more severe or complex casemix but also factors such as processes of care, administrative delays around organising tests and discharge, and the availability of community rehabilitation and care homes. It is notable that within England, the same region with the longest stays and total time spent in hospital has in fact the lowest mortality (London): this matches the difference between the two countries, with both London and Lombardy having longer average stays, greater total in-hospital time and lower in-hospital mortality than England as a whole. Secondly, patients aged 85 had both shorter stays and longer stays in England than in Lombardy, as seen in their low lower quartile and high upper quartile and 90th percentiles. Pressure on acute inpatient beds can lead to some patients being prematurely discharged - it has been previously shown in England that same-day discharges have a higher 30-day readmission rate than those staying one or more nights [23]. At the same time, the phenomenon of "delayed transfers of care" has been increasingly seen in the NHS in recent years, with patients who are deemed ready for discharge having to wait days or weeks in hospital for residential care. The latter issue will affect the very elderly more than the young, and both issues could help explain the LOS distribution differences between the countries.

For all of the apparent differences between the countries, clinical practice and quality is also a potential explanation and of course is the most interesting one. The European Society of Cardiology international registry, based on a small sample of centres in each country, found variations in clinical features, management and mortality between world regions, with Southern Europe having the lowest one-year mortality [20]. The big question is whether the greater time spent in hospital in Lombardy (and London) leads to better survival in the short and medium term and other health benefits. The extra admissions per person in Lombardy offer more opportunities for the team to apply guideline-based medications, revascularization and device-based therapy and also educate the patient, thereby improving survival, at least in the short-term. For this reason, some commentators have argued that not all readmissions are bad [24]. The National Heart Failure Audit for England and Wales reports annually on hospital outcomes and processes such as prescribing and cardiologist input, but there is no equivalent for Italy to allow us to compare quality directly. The England and Wales Audit has shown falls in in-hospital and one-year mortality and concomitant improvements in evidence-based standards such as prescribing and specialist input over time [25]. It also reported that the average length of stay in hospital was longer and the mortality rate lower for patients on cardiology wards than on medical wards. This is consistent with the claim that specialist cardiology clinicians spend more time optimising therapies and ensuring stability prior to discharge, resulting in longer hospital admissions. We believe that one plausible explanation of our findings is that, compared with England, in Lombardy there is a greater willingness to admit patients for their HF and to keep them in hospital longer in order to better tune their therapies and so reduce mortality. The greater proportion of very long stays among the very elderly in England's NHS is more likely due to problems with appropriate discharge than with better care. However, ours is an observational study, and so causal inference is not possible regarding which of the competing explanations for the differences is true.

\subsection{Strengths and limitations}

This study benefitted from large sample sizes drawn from databases with regional or national coverage, common advantages with administrative data. As described earlier, multistate models have some key benefits over standard logistic regression for 30-day readmission or survival analysis for single or multiple events. These include handling both the competing risk of death and the serial nature of admissions due to disease progression. They are within an inferential framework and are therefore more powerful than using only the simple descriptive analyses that we presented first. In particular, they allow for prediction.

We have assumed that the first unplanned admission for the patient's HF represents a significant deterioration in the patient's health status. This index HF admission is an often-used and convenient starting point from which to examine their subsequent admission trajectory, but for cardiologists it represents an important milestone in the disease progression at which further intervention is needed and the management changes. For the patient it means their symptoms have worsened and of course their chance of death at this point is notably raised.

As the Lombardy data came from a demonstration project, we did not have non-HF admissions or a flag for diabetes without complications for that region. Patients with HF usually have multiple comorbidities and are admitted more for these conditions than for HF [23]. Some quality of care measures and some predictors of readmission are associated only with HF readmissions but not with other readmissions [26]. Administrative databases typically have some underrecording of comorbidities, though this has been found not to bias 
estimated relations between comorbidity and mortality [27] and the primary diagnosis is well recorded [22]. We were unable to capture symptom severity or other physiological parameters for either country, as these are not included in the databases.

To enable convergence with the msm package, we had to restrict the set of comorbidities to the commonest ones and limit the number of states to eight, allowing for the first three admissions to be considered separately.

\section{Conclusion}

This study revealed considerable apparent differences between England and Lombardy in mortality and HF readmission patterns. Such studies are useful for generating hypotheses relating to possible mechanisms for these differences, as we are unable to confidently disentangle the impact of coding, casemix, clinical practice, and the availability and use of nonhospital settings. Future work on such cross-country comparisons in patients with heart failure should investigate admissions for non-HF diagnoses and the effect of other comorbidities on disease progression. We believe that multistate models would be a useful component of such work.

\begin{abstract}
Acknowledgements The Dr. Foster Unit at Imperial College London is partially funded by a grant from Dr. Foster ${ }^{\circledR}$, a private healthcare information company. The Dr. Foster Unit at Imperial is affiliated with the National Institute of Health Research (NIHR) Imperial Patient Safety Translational Research Centre. The NIHR Imperial Patient Safety Translational Centre is a partnership between the Imperial College Healthcare NHS Trust and Imperial College London. The Dr. Foster Unit at Imperial College are grateful for support from the NIHR Biomedical Research Centre funding scheme. The Dr. Foster Unit at Imperial College London is partly funded by research grants from the National Institute for Health Research Health Services Research. Dr. Dharmarajan is supported by grant K23AG048331 from the National Institute on Aging and the American Federation for Aging Research through the Paul B. Beeson Career Development Award Program. He is also supported by grant P30AG021342 via the Yale Claude D. Pepper Older Americans Independence Center. The views and opinions expressed herein are those of the authors and do not necessarily reflect those of the NHS, the NIHR, Medical Research Council, Central Commissioning Facility, NETSCC, the Health Services and Delivery Research programme or the Department of Health.
\end{abstract}

\section{Compliance with ethical standards}

Ethics We have approval from the Secretary of State and the Health Research Authority under Regulation 5 of the Health Service (Control of Patient Information) Regulations 2002 to hold confidential data and analyse them for research purposes (CAG ref 15/CAG/0005). We have approval to use them for research and measuring quality of delivery of healthcare, from the London - South East Ethics Committee (REC ref 15/LO/0824).

Open Access This article is distributed under the terms of the Creative Commons Attribution 4.0 International License (http:// creativecommons.org/licenses/by/4.0/), which permits unrestricted use, distribution, and reproduction in any medium, provided you give appropriate credit to the original author(s) and the source, provide a link to the Creative Commons license, and indicate if changes were made.

\section{References}

1. GBD (2015) Disease and injury incidence and prevalence collaborators (2016) global, regional, and national incidence, prevalence, and years lived with disability for 310 diseases and injuries, 19902015: a systematic analysis for the global burden of disease study 2015. Lancet 388:1545-1602

2. Roger VL (2013) Epidemiology of heart failure. Circ Res 113:646-659

3. van Riet EES, Hoes A, Wagenaar KP, Limburg A, Landman MAJ, Rutten FH (2016) Epidemiology of heart failure: the prevalence of heart failure and ventricular dysfunction in older adults over time. A systematic review. Eur J Heart Fail 18:242-252

4. Eurostat (2016) Healthcare resource statistics - beds. Available at URL: http://ec.europa.eu/eurostat/statistics-explained/index.php/ Healthcare_resource_statistics_-_beds. Accessed 21 Nov 2016

5. Regione Lombardia (2016) Lombardy healthcare system. Available at URL: http://www.regione.lombardia.it/cs/ Satellite $? \mathrm{c}=\mathrm{Page} \& \mathrm{child}$ agename $=$ Regione $\% 2 \mathrm{FPage} \%$ 2FENMILayout\&cid=1213583535815\&pagename $=$ RGNWrapper. Accessed 21 Nov 2016

6. Brenna E. (2016) The Lombardy health care system. Working Paper. Available at URL: http://www.unicatt.it/Istituti/ EconomiaImpresaLavoro/Quaderni/ieil0063.pdf. Accessed 24 Nov 2016

7. Bevan G, Karanikolos M, Exley J, Nolte E, Connolly S, Mays N (2014) The four health systems of the United Kingdom: how do they compare? Health Foundation and the Nuffield Trust 2014. Available from URL: http://www.nuffieldtrust.org.uk/sites/files/ nuffield/revised_4_countries_report.pdf. Accessed 24 Nov 2016

8. Dharmarajan K, Hsieh AF, Kulkarni VT, Lin Z, Ross RS, Horwitz LI, Kim N, Suter LG, Lin H, Normand S-LT, Krumholz HM (2015) Trajectories of risk after hospitalization for heart failure, acute myocardial infarction, or pneumonia: retrospective cohort study. BMJ 350:h411

9. Chun S, Tu JV, Wijeysundera HC, Austin PC, Wang X, Levy D, Lee DS (2012) Lifetime analysis of hospitalizations and survival of patients newly admitted with heart failure. Circ Heart Fail 5: 414-421

10. van Walraven C, Dhalla IA, Bell C, Etchells E, Stiell IG, Zarnke K, Austin PC, Forster AJ (2010) Derivation and validation of an index to predict early death or unplanned readmission after discharge from hospital to the community. CMAJ 182(6):551-557

11. Bottle A, Gaudoin R, Goudie R, Jones S, Aylin P (2014) Can valid and practical risk-prediction or casemix adjustment models, including adjustment for comorbidity, be generated from English hospital administrative data (hospital episode statistics)? A national observational study. Health Serv Deliv Res 2(40)

12. Ieva F, Jackson CH, Sharples LD (2017) Multi-state modelling of repeated hospitalisation and death in patients with heart failure: the use of large administrative databases in clinical epidemiology. Stat Methods Med Res 26(3):1350-1372

13. Bartolomeo N, Trerotoli P, Moretti A, Serio G (2008) A Markov model to evaluate hospital readmission. BMC Med Res Methodol 8:23

14. Postmus D, van Veldhuisen DJ, Jaarsma T, Luttik ML, Lassus J, Mebazaa A, Nieminen MS, Harjola VP, Lewsey J, Buskens E, Hillege HL (2012) The COACH risk engine: a multistate model 
for predicting survival and hospitalization in patients with heart failure. Eur J Heart Fail 14(2):168-175

15. Bakal JA, McAlister FA, Liu W, Ezekowitz JA (2014) Heart failure re-admission: measuring the ever shortening gap between repeat heart failure hospitalizations. PloS One 9(9):e106494

16. OECD (2015) OECD reviews of health care quality: Italy 2014: raising standards. OECD Publishing, Paris

17. Mazzali C, Paganoni AM, Ieva F, Masella C, Maistriello M, Agostoni O, Scalvini S, Frigerio M (2016) Methodological issues on the use of administrative data in healthcare research: the case of heart failure hospitalizations in Lombardy region, 2000 to 2012. BMC Health Serv Res 16(1):234

18. Jackson C (2011) Multi-state models for panel data: the msm package for R. J Stat Softw 38(8)

19. Meira-Machado L, de Uña-Álvarez J, Cadarso-Suárez C, Andersen PK (2009) Multi-state models for the analysis of time-to-event data. Stat Methods Med Res 18(2):195-222

20. Bhatnagar P, Wickramasinghe K, Williams J, Rayner M, Townsend N (2015) The epidemiology of cardiovascular disease in the UK 2014. Heart 101(15):1182-1189

21. Crespo-Leiro MG, Anker SD, Maggioni AP, Coats AJ, Filippatos G, Ruschitzka F, Ferrari R, Piepoli MF, Delgado Jimenez JF, Metra M, Fonseca C, Hradec J, Amir O, Logeart D, Dahlström U, Merkely B, Drozdz J, Goncalvesova E, Hassanein M, Chioncel O, Lainscak M, Seferovic PM, Tousoulis D, Kavoliuniene A,
Fruhwald F, Fazlibegovic E, Temizhan A, Gatzov P, Erglis A, Laroche C, Mebazaa A, Heart Failure Association (HFA) of the European Society of Cardiology (ESC) (2016) European Society of Cardiology Heart Failure Long-Term Registry (ESC-HF-LT): 1year follow-up outcomes and differences across regions. Eur J Heart Fail 18(6):613-625

22. Burns EM, Rigby E, Mamidanna R, Bottle A, Aylin P, Ziprin P, Faiz OD (2011) Systematic review of discharge coding accuracy. J Public Health 34(1):138-148

23. Bottle A, Aylin P, Bell D (2014) Effect of the readmission primary diagnosis and time interval in heart failure patients: analysis of English administrative data. Eur J Heart Fail 16(8): 846-853

24. Vaduganathan M, Bonow RO, Gheorghiade M (2013) Thirty-day readmissions: the clock is ticking. JAMA 309(4):345-346

25. Cleland J, Dargie H, Hardman S, McDonagh T, Mitchell P (2013) National Heart Failure Audit April 2012-March 2013. National Institute for Cardiovascular Outcomes Research

26. Bottle A, Goudie R, Cowie MR, Bell D, Aylin P (2015) Relation between process measures and diagnosis-specific readmission rates in patients with heart failure. Heart 101(21):1704-1710

27. Quan H, Parsons GA, Ghali WA (2002) Validity of information on comorbidity derived from ICD-9-CCM administrative data. Med Care 40:675-685 